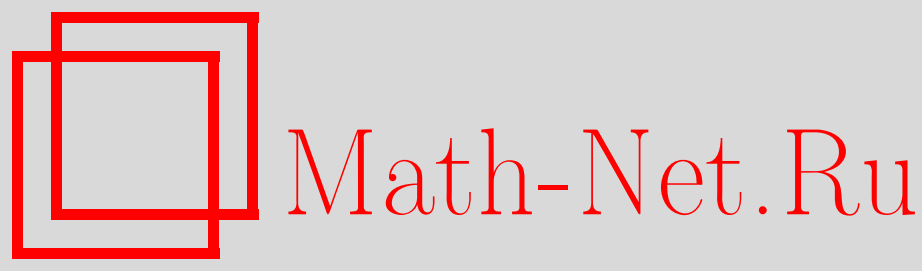

А. В. Маршаков, Неабелевы калибровочные теории, препотенциалы и абелевы дифференциалы, ТМФ, 2009, том 159, номер 2, 220-242

DOI: https://doi.org/10.4213/tmf6344

Использование Общероссийского математического портала Math-Net.Ru подразумевает, что вы прочитали и согласны с пользовательским соглашением http: //www . mathnet.ru/rus/agreement

Параметры загрузки:

IP: 54.198 .67 .100

26 апреля 2023 г., 18:08:02

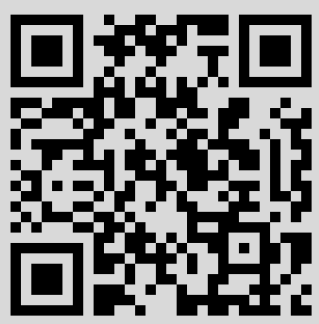




\title{
НЕАБЕЛЕВЫ КАЛИБРОВОЧНЫЕ ТЕОРИИ, ПРЕПОТЕНЦИАЛЫ И АБЕЛЕВЫ ДИФФЕРЕНЦИАЛЫ
}

\begin{abstract}
Обсуждаются специальные решения интегрируемых систем начиная с хорошо известных бездисперсионных иерархий Кортевега-де Фриза и Тоды, которые определяют наиболее простым способом производящие функции для классов Громова-Виттена в терминах рациональной комплексной кривой. С точки зрения зеркальной теории эти производящие функции можно отождествить с препотенциалами комплексных многообразий. Приводятся несколько новых явно вычисляемых примеров таких препотенциалов. Для кривых старших родов, отвечающих в данном контексте неабелевым калибровочным теориям в рамках дуальности между топологическими струнами и калибровочными теориями, аналогичные решения строятся с использованием расширенного базиса абелевых дифференциалов, включающего, вообще говоря, дифференциалы с особенностями в точках ветвления кривой.
\end{abstract}

Ключевые слова: суперсимметричные калибровочные теории, топологические струны, интегрируемые системы.

\section{1. ВВЕДЕНИЕ}

Интегрируемые дифференциальные уравнения возникают в различных разделах современной математической физики, но в последние годы они привлекают особенное внимание в связи с изучением статсумм в простейших моделях теории струн и некоторых квантовых теориях поля. Производящие функции для корреляторов, которые символически можно записать в виде

$$
\ln \tau(\mathbf{t})=\left\langle\exp \sum_{k} t_{k} \sigma_{k}\right\rangle_{\text {string }},
$$

определяются изначально суммированием рядов теории возмущений и даже инстантонных вкладов в формулировке моделей теории струн на мировых поверхностях и в достаточно простых квантовых теориях поля. Оказывается, однако, что вся существенная информация о результатах этого суммирования обычно спрятана в системах достаточно простых (хотя и, вообще говоря, нелинейных) дифференциальных уравнений на функцию $\ln \tau(\mathbf{t})$ и ее производные.

${ }^{*}$ Физический институт им. П. Н. Лебедева РАН; Институт теоретической и экспериментальной физики, Москва, Россия. E-mail: mars@lpi.ru, mars@itep.ru 
В некотором смысле физикам-теоретикам повезло: как для простейших модельных примеров, так и для некоторых физических теорий в многомерном пространстве-времени возникают хорошо знакомые и широко используемые в прикладных задачах математической физики интегрируемые системы в размерностях $1+1$ и $2+1$. Более того, часто оказываются интересными их решения с конечным числом степеней свободы - так называемые модули теории (отвечающие конечному набору примарных операторов и соответствующих временных параметров, иногда также называемых "малым фазовым пространством"). Геометрия таких решений базируется в основном на комплексных кривых и их якобианах, так что отображение Абеля играет роль интегрирующей замены переменных.

Будучи тесно связанными с алгебро-геометрическими решениями интегрируемых систем типа Кадомцева-Петвиашвили (КП) и Тоды, струнные решения являются, тем не менее, абсолютно новыми. Лучший способ продемонстрировать это - начать с двух хорошо известных примеров.

Пример 1. Знаменитое уравнение Кортевега-де Фриза $\left(\right.$ КдФ) $u_{t}+u u_{x}+u_{x x x}=0$ тривиально удовлетворяется для функции $u=x / t$, представляющей собой "медленно опускающийся" с течением времени линейный потенциал. Это достаточно тривиальное решение с точки зрения самого уравнения КдФ отвечает чистой топологической гравитации (простейшей топологической струнной модели) или теории Громова-Виттена для точки [1]-[4], для которой статсумма (известная также как модель Концевича)

$$
\begin{aligned}
\ln \tau & =\mathrm{F}_{\mathrm{K}}(x, t)+\cdots=\frac{x^{3}}{6 t}+\cdots \underset{t \rightarrow t+1}{=} \frac{x^{3}}{3 !}\langle\mathbf{1 1 1}\rangle+\cdots, \\
u & =\frac{\partial^{2} \ln \tau}{\partial x^{2}}
\end{aligned}
$$

генерирует числа пересечений

$$
\left\langle\sigma_{k_{1}} \ldots \sigma_{k_{n}}\right\rangle=\int_{\bar{M}_{g, n}} \prod_{i=1}^{n} \psi_{i}^{k_{i}}
$$

определенные как интегралы по компактифицированным пространствам модулей $\bar{M}_{g, n}$ комплексных кривых рода $g$ с $n$ отмеченными точками (мировых листов для $n$-точечных корреляторов в теории струн), где $\psi_{i}=c_{1}\left(\mathbb{L}_{i}\right)$ является первым классом Чженя канонического линейного расслоения над $\bar{M}_{g, n}$ со слоем $T_{\Sigma_{g, n}}^{*}\left(P_{i}\right)$ в $i$-й отмеченной точке $P_{i}$. В формуле (2) выписано только единственное число $\langle\mathbf{1 1 1}\rangle=1$, отвечающее тривиальному интегралу по $\bar{M}_{0,3}=$ point. Чтобы найти остальные числа пересечений (3), следует решить полную иерархию уравнения КдФ (включить потоки по остальным старшим переменным иерархии $t_{1}=x$, $\left.t_{3}=t, t_{5}, t_{7}, \ldots\right)$ с начальным условием $u=x / t$

$$
\ln \tau=\sum_{\left\{k_{i}\right\} \geqslant 0} \frac{t_{2 k_{1}+1} \ldots t_{2 k_{n}+1}}{n !}\left\langle\sigma_{k_{1}} \ldots \sigma_{k_{n}}\right\rangle \hbar^{2 g-2} .
$$


На физическом языке формулой (4) задается производящая функция корреляторов гравитационных потомков $\sigma_{k} \equiv \sigma_{k}(\mathbf{1})$, отвечающих умножению примарных операторов на $k$-е степени классов Чженя. "Пространственно-временная" часть теории для точки тривиальна и содержит единственный единичный примарный оператор 1. Для удобства в формуле (4) явно введена струнная константа связи $\hbar$ с весом, который фиксируется правилом отбора $\sum k_{i}=3 g-3+n$. Например, вклад, явно выписанный в формуле (2), умножается на $\hbar^{-2}$, так как он отвечает пространству модулей $\bar{M}_{0,3}$ с $g=0$.

Этот дополнительный параметр крайне полезен, так как специальный интерес представляет квазиклассический предел $\hbar \rightarrow 0$ производящей функции, с поведением

$$
\ln \tau(\mathbf{t}) \underset{\hbar \rightarrow 0}{\sim} \frac{1}{\hbar^{2}} \mathcal{F}(\mathbf{t})+O\left(\hbar^{0}\right),
$$

где $\mathcal{F}$ часто называют препотенциалом. Квазиклассическая часть разложения (4) описывается в терминах бездисперсионного предела иерархии КдФ с функцией Лакса $W=z^{2}-u$ (оператор Лакса после подстановки $\left.\partial / \partial x \rightarrow \hbar \partial / \partial x \rightarrow z\right)$, или явным образом с помощью формул

$$
\begin{aligned}
x & =\left.\operatorname{res}\right|_{z=\infty} W^{-1 / 2} z d W \sim u, \\
\frac{\partial \mathcal{F}}{\partial x} & =\left.\operatorname{res}\right|_{z=\infty} W^{1 / 2} z d W \sim u^{2},
\end{aligned}
$$

так что

$$
\mathcal{F} \underset{\hbar \rightarrow 0}{=} \hbar^{2} \ln \tau=\mathrm{F}_{\mathrm{K}}(x) \sim x^{3}+\cdots .
$$

Формулы типа (6) определяют препотенциал (7) или квазиклассическую часть (4) как вырожденный препотенциал почти тривиального комплексного многообразия или пространственно-временной рациональной кривой $W=z^{2}-u$. В общем случае имеется много переменных, вычеты должны быть заменены на интегралы периодов, res $\rightarrow \oint$, по всем нетривиальным циклам, а интегрируемость уравнений типа (6) обеспечивается билинейными соотношениями Римана.

Полная производящая функция $\tau(\mathbf{t})$ может быть также восстановлена [5] как решение условий Вирасоро

$$
\begin{gathered}
L_{n} \tau=0, \quad n \geqslant-1, \\
L_{n}=\frac{1}{2} \sum k t_{k} \frac{\partial}{\partial t_{k+2 n}}+\frac{1}{4} \sum_{a+b=2 n} \frac{\partial^{2}}{\partial t_{a} \partial t_{b}}+\delta_{n+1,0} \frac{t_{1}^{2}}{4}+\frac{\delta_{n, 0}}{16},
\end{gathered}
$$

представляющих собой бесконечную систему линейных дифференциальных уравнений.

ПримеР 2. Перейдем теперь ко второму простейшему примеру цепочки Тоды [6] (в бездисперсионном пределе):

$$
\frac{\partial^{2} \mathcal{F}}{\partial t_{1}^{2}}=e^{\partial^{2} \mathcal{F} / \partial a^{2}}
$$


Струнное решение

$$
\mathcal{F}=\frac{1}{2} a^{2} t_{1}+e^{t_{1}}
$$

(снова для препотенциала $\mathcal{F} \underset{\hbar \rightarrow 0}{=} \hbar^{2} \ln \tau$ ) описывает систему частиц с координатами $a^{D}=\partial \mathcal{F} / \partial a=a t_{1}$, движущихся с постоянными скоростями, равными их номерам $a$, во времени $t_{1}$, являющемся первым временем иерархии цепочки Тоды. Эти два параметра $\left(a, t_{1}\right)$ заменяют здесь единственный пространственно-временной параметр $x$ иерархии КдФ, так как примарные операторы теперь (вместо единственного оператора 1 в примере 1) отвечают когомологиям $\mathbb{P}^{1}: a \leftrightarrow \mathbf{1} \in H^{0}\left(\mathbb{P}^{1}\right)$ и $t_{1} \leftrightarrow \varpi \in H^{2}\left(\mathbb{P}^{1}\right)$. Редуцированная производящая функция $\mathcal{F} \sim\left\langle e^{a \mathbf{1}+t_{1} \varpi}\right\rangle$ приводит к деформации умножения в кольце когомологий: $\varpi \cdot \varpi \simeq e^{t_{1}} \mathbf{1}$, отвечающему единственному нетривиальному соотношению в операторной алгебре пространственно-временных примарных операторов.

Чтобы восстановить зависимость от гравитационных потомков $t_{k+1} \leftrightarrow \sigma_{k}(\varpi)$, $T_{n} \leftrightarrow \sigma_{n}(\mathbf{1})$ (в этих обозначениях $a \equiv-T_{0}$ ), следует найти решение иерархии цепочки Тоды с начальным условием, отвечающим решению (10). В квазиклассическом приближении для препотенциала

$$
\mathcal{F}=\frac{a^{2} t_{1}}{2}+e^{t_{1}} \Rightarrow \mathcal{F}(\mathbf{t}, a) \Rightarrow \mathcal{F}(\mathbf{t}, \mathbf{T})
$$

это может быть сделано в два шага (конечно, и наполовину редуцированная производящая функция $\mathcal{F}(\mathbf{t}, a)$, и полная функция $\mathcal{F}(\mathbf{t}, \mathbf{T})$ по-прежнему удовлетворяют первому уравнению Тоды (9)). Решение иерархии цепочки Тоды по переменным $\mathbf{t}$ приводит к (наполовину редуцированной) теории Громова-Виттена комплексной проективной прямой $\mathbb{P}^{1}$

$$
\ln \tau=\sum_{\left\{k_{i}\right\}, d \geqslant 0} \frac{t_{k_{1}} \ldots t_{k_{n}}}{n !}\left\langle\sigma_{k_{1}}(\varpi) \ldots \sigma_{k_{n}}(\varpi)\right\rangle \hbar^{2 g-2} q^{d}
$$

где $\sigma_{k}(\varpi)$ представляют собой потомки (вообще говоря, комплексифицированного) кэлерова класса $\varpi$ (чтобы отличать их от потомков $\sigma_{k}(\mathbf{1})$ единичного оператора), а корреляторы теперь отождествляются с интегралами по пространствам модулей $\bar{M}_{g, n}\left(\mathbb{P}^{1}, d\right)$ стабильных отображений степени $d$ кривых рода $g$ с $n$ отмеченными точками в проективную прямую $\sum_{g, n} \mapsto \mathbb{P}^{1}$ :

$$
\left\langle\sigma_{k_{1}} \ldots \sigma_{k_{n}}\right\rangle=\int_{\bar{M}_{g, n}(\mathbb{P} 1, d)} \prod_{i=1}^{n} \psi_{i}^{k_{i}} \operatorname{ev}_{i}^{*}(\varpi),
$$

где оператор $\mathrm{ev}_{i}: \bar{M}_{g, n}\left(\mathbb{P}^{1}, d\right) \mapsto \mathbb{P}^{1}$ представляет собой вычисление отображения в $i$-й отмеченной точке. От дополнительного параметра $q$ в формуле $(12)$, считающего степени отображений или инстантонный заряд, можно избавиться с помощью сдвига $t_{1} \rightarrow t_{1}-\ln q$.

Квазиклассическая часть производящей функции (12) опять описывается препотенциалом в дуальной картине, иногда называемой подходом Гинзбурга-Ландау. 
Суперпотенциал Гинзбурга-Ландау может быть выбран как функция на цилиндре

$$
z=v+\Lambda\left(w+\frac{1}{w}\right)
$$

имеющая также очевидный смысл функции Лакса для бездисперсионной цепочки Тоды (правая часть равенства (14) представляет собой трехдиагональную матрицу Лакса цепочки Тоды в терминах степеней спектрального параметра $w)$. Уравнение (14) можно рассматривать как описывающее рациональную кривую (цилиндр), помещенную в двумерное комплексное пространство, $(z, w) \subset \mathbb{C} \times \mathbb{C}^{*}$, и его можно также считать частным случаем семейства кривых $N_{c}$-периодической цепочки Тоды

$$
\Lambda^{N_{c}}\left(w+\frac{1}{w}\right)=P_{N_{c}}(z)=\prod_{i=1}^{N_{c}}\left(z-v_{i}\right) .
$$

Топологическая струна типа $\mathrm{A}$ на $\mathbb{P}^{1}$, таким образом, является дуальной $N_{c}=1$ упрощенной абелевой $\mathcal{N}=2$ суперсимметричной калибровочной теории [7]. Поэтому решение бездисперсионной цепочки Тоды, отвечающей кривой (14), является промежуточным шагом к пониманию геометрии расширенной неабелевой $\mathcal{N}=2$ суперсимметричной калибровочной теории с калибровочной группой $U\left(N_{c}\right)$, обычно ассоциируемой в контексте теории Виттена-Зайберга [8] с семейством кривых (15).

\section{2. ТОПОЛОГИЧЕСКОЕ РЕШЕНИЕ БЕЗДИСПЕРСИОННОЙ ИЕРАРХИИ ТОДЫ}

Решение наполовину редуцированной бездисперсионной иерархии цепочки Тоды для $\mathcal{F}(\mathbf{t}, a)$ при $T_{n}=\delta_{n, 1}$, или выключенных гравитационных потомках единичного оператора $\left\{\sigma_{k}(\mathbf{1})\right\}$, задается в терминах рациональной кривой (14) (или оператора Лакса бездисперсионной цепочки Тоды), оснащенной функцией с асимптотикой

$$
S \underset{z \rightarrow \infty}{=}-2 z(\ln z-1)+\sum_{k>0} t_{k} z^{k}+2 a \ln z-\frac{\partial \mathcal{F}}{\partial a}-2 \sum_{k>0} \frac{1}{k z^{k}} \frac{\partial \mathcal{F}}{\partial t_{k}},
$$

нечетной относительно инволюции $w \leftrightarrow 1 / w$, которая имеет смысл логарифма функции $\Psi$, решающей вспомогательную линейную задачу. Как обычно в интегрируемых системах, решения для самих динамических переменных возникают из построения функций $\Psi$ или $S$. В терминах глобальной переменной $w$ немедленно имеем [9]

$$
S=-2\left(z \ln w+\Lambda(\ln \Lambda-1)\left(w-\frac{1}{w}\right)\right)+\sum_{k>0} t_{k} \Omega_{k}(w)+2 a \ln w
$$

что полностью фиксируется асимптотикой при $w \underset{z \rightarrow \infty}{\sim} z$ и нечетностью относительно инволюции $w \leftrightarrow 1 / w$. Здесь $\Omega_{k}=z(w)_{+}^{k}-z(w)_{-}^{k}$, где знаки "+" и "-" отвечают строго положительной и отрицательной частям полиномов Лорана (степеням $z$ из (14)) от переменной $w$, например $\Omega_{1}(w)=\Lambda(w-1 / w), \Omega_{2}(w)=\Lambda^{2}\left(w^{2}-1 / w^{2}\right)+2 \Lambda v(w-1 / w)$ и т.д. 
Выражения для $v, \Lambda, \mathcal{F}$ как функций $a$ и всех времен $\mathbf{t}$ находятся из условий

$$
\left.\frac{d S}{d \ln w}\right|_{d z=0}=0
$$

наложенных в нулях дифференциала $d S$, совпадающих с нулями $d z$, т.е. в точках ветвления [10]. Они представляют собой два алгебраических уравнения, решаемых относительно коэффициентов $v=v(a ; \mathbf{t})$ и $\Lambda=\Lambda(a ; \mathbf{t})$ кривой $(14)$.

2.1. Малое фазовое пространство. Если, например, $t_{k}=0$ при $k>1$, имеем

$$
v=a, \quad \Lambda^{2}=e^{t_{1}},
$$

а из "регулярного хвоста" разложения (16) считывается препотенциал (10) на малом фазовом пространстве. Эту операцию можно интерпретировать как особенный вырожденный случай общего построения препотенциала комплексной кривой $\Sigma$, оснащенной двумя мероморфными дифференциалами с фиксированными периодами [10] или производящей 1-формой Виттена-Зайберга $d S_{\mathrm{SW}}$. Переменные в общем случае вводятся как интегралы периодов

$$
a_{i}=\frac{1}{4 \pi i} \oint_{A_{i}} d S_{\mathrm{SW}}
$$

по выбранной половине циклов в $H_{1}(\Sigma)$, а градиенты препотенциала $\mathcal{F}$ определяются интегралами периодов по дуальным циклам:

$$
a_{i}^{D}=\oint_{B_{i}} d S_{\mathrm{SW}}=\frac{\partial \mathcal{F}}{\partial a_{i}} .
$$

Определение (21) является самосогласованым благодаря условию

$$
\frac{\partial a_{i}^{D}}{\partial a_{j}}=T_{i j}=\frac{\partial^{2} \mathcal{F}}{\partial a_{i} \partial a_{j}},
$$

где симметричность правой части равенства (или вторых производных препотенциала) обеспечивается симметричностью матрицы периодов кривой $\Sigma$. Равенство (22) следует из (21) при том условии, что вариация дифференциала $d S_{\mathrm{SW}}$ по модулям голоморфна, что является прямым аналогом свойства (18).

Приведенное выше решение бездисперсионной цепочки Тоды с единственными ненулевыми переменными $a$ и $t_{1}$ представляет собой просто вырожденный случай этой конструкции, когда вместо нетривиальной кривой мы взяли цилиндр. Тогда с учетом (19)

$$
\begin{aligned}
S & =-2\left(z \ln w+\Lambda(\ln \Lambda-1)\left(w-\frac{1}{w}\right)\right)+t_{1} \Lambda\left(w-\frac{1}{w}\right)+2 a \ln w= \\
& =-2 z \ln w+2 \Lambda\left(w-\frac{1}{w}\right)+2 a \ln w, \quad d S=-2 d z \ln w, \quad d S_{\mathrm{SW}}=2 z \frac{d w}{w}
\end{aligned}
$$

так что

$$
\frac{1}{4 \pi i} \oint_{A_{i}} d S_{\mathrm{SW}}=\frac{1}{2 \pi i} \oint_{A} z \frac{d w}{w}=\left.\operatorname{res}\right|_{z=\infty} z \frac{d w}{w}=a
$$


$(d z$ и $d w / w$ как раз и являются двумя мероморфными дифференциалами с фиксированными периодами), и

$$
\frac{\partial \mathcal{F}}{\partial a} \sim \int_{B} z \frac{d w}{w} \sim[S]_{0}=a t_{1},
$$

где роль регуляризованного бесконечного вырожденного $B$-периода играет постоянная часть функции (16).

2.2. Старшие потоки. Чтобы добавить старшие потоки, следует ввести обобщенные периоды, или просто коэффициенты разложения (16), которые можно записать как

$$
t_{k}=\left.\frac{1}{k} \operatorname{res}\right|_{z=P_{+}} z^{-k} d S=-\left.\frac{1}{k} \operatorname{res}\right|_{z=P_{-}} z^{-k} d S, \quad k>0
$$

и

$$
\frac{\partial \mathcal{F}}{\partial t_{k}}=\left.\frac{1}{2} \operatorname{res}\right|_{z=P_{+}} z^{k} d S=-\left.\frac{1}{2} \operatorname{res}\right|_{z=P_{-}} z^{k} d S, \quad k>0,
$$

где $z\left(P_{+}\right)=z\left(P_{-}\right)=\infty$ и эти точки представляют собой две бесконечности на кривой (14), меняющиеся друг с другом при действии инволюции $w \leftrightarrow 1 / w$. Уравнения (18) остаются теми же, но в общем случае их нельзя решить явным образом. Уже добавив лишь ненулевое $t_{2}$, решения уравнений (18) [9]

$$
\begin{aligned}
v & =a-\frac{1}{2 t_{2}} \mathbf{L}\left(-4 t_{2}^{2} e^{t_{1}+2 t_{2} a}\right), \\
\ln \Lambda^{2} & =t_{1}+2 t_{2} a-\mathbf{L}\left(-4 t_{2}^{2} e^{t_{1}+2 t_{2} a}\right)
\end{aligned}
$$

можно выразить только через функцию Ламберта $\mathbf{L}(x) e^{\mathbf{L}(x)}=x$. Это не что иное, как асимптотика производящей функции для чисел Гурвица

$$
H_{g, d}=\left\langle\sigma_{1}(\varpi)^{2 g+2 d-2}\right\rangle_{g, d},
$$

каждое из которых имеет смысл числа накрытий рода $g$, с $d$ листами проективной прямой $\mathbb{P}^{1}$, с фиксированным общим дивизором точек ветвления степени $d \cdot \chi\left(\mathbb{P}^{1}\right)-$ $\chi\left(\Sigma_{g}\right)=2 d+2 g-2$, как следует из формулы Римана-Гурвица.

В самом деле, из (12), (29) имеем

$$
\mathcal{F}\left(a=0, t_{1}, t_{2}=\frac{1}{2}, 0, \ldots\right)=\sum_{d>0} \frac{H_{d, 0}}{(2 d-2) !} e^{d t_{1}} .
$$

Из решения (28) следует

$$
\left.\frac{\partial^{2} \mathcal{F}}{\partial t_{1}^{2}}\right|_{a=0, t_{2}=1 / 2}=\Lambda^{2}=-\mathbf{L}\left(-e^{t_{1}}\right),
$$

что дает буквально $H_{d, 0}=(2 d-2) ! d^{d-3} / d$ !, так как функция Ламберта имеет разложение

$$
\mathbf{L}(t)=\sum_{n=1}^{\infty} \frac{(-n)^{n-1} t^{n}}{n !}=t-t^{2}+\frac{3}{2} t^{3}-\frac{8}{3} t^{4}+\cdots,
$$

непосредственно приводящее к нужному результату. 


\section{3. КВАЗИКЛАССИЧЕСКОЕ ПРИБЛИЖКННИЕ СТАТСУММЫ НЕКРАСОВА}

Тау-функция (12) может быть определена вне рамок квазиклассического (отвечающего потенциалу Громова-Виттена рода $g=0)$ приближения [11], [7]. Ее определение дается на языке суммы по разбиениям: по наборам целых чисел $\mathbf{k}=\left(k_{1} \geqslant\right.$ $\left.k_{2} \geqslant \cdots \geqslant k_{\ell_{\mathbf{k}}}=0 \geqslant 0 \cdots\right)$

$$
\tau(a, \mathbf{t})=\sum_{\mathbf{k}} \frac{\mathbf{m}_{\mathbf{k}}^{2}}{\left(-\hbar^{2}\right)^{|\mathbf{k}|}} \exp \left[\frac{1}{\hbar^{2}} \sum_{k>0} \frac{t_{k}}{k+1} \operatorname{Ch}_{k+1}(a, \mathbf{k}, \hbar)\right] \sim \exp \left(\frac{1}{\hbar^{2}} \mathcal{F}(a, \mathbf{t})+\cdots\right)
$$

с весами, пропорциональными квадрату меры Планшереля

$$
\mathbf{m}_{\mathbf{k}}=\frac{\prod_{1 \leqslant i<j \leqslant \ell_{\mathbf{k}}}\left(k_{i}-k_{j}+j-i\right)}{\prod_{i=1}^{\ell_{\mathbf{k}}}\left(\ell_{\mathbf{k}}+k_{i}-i\right) !} \sim \prod_{i<j} \frac{k_{i}-k_{j}+j-i}{j-i}
$$

и зависящими от тодовских времен с помощью полиномов Чженя

$$
\left(e^{\hbar u / 2}-e^{-\hbar u / 2}\right) \sum_{i=1}^{\infty} e^{u\left(a+\hbar\left(1 / 2-i+k_{i}\right)\right)}=\sum_{l=0}^{\infty} \frac{u^{l}}{l !} \operatorname{Ch}_{l}(a, \mathbf{k} ; \hbar)
$$

или

$$
\begin{aligned}
& \mathrm{Ch}_{0}(a, \mathbf{k})=1, \quad \mathrm{Ch}_{1}(a, \mathbf{k})=a, \\
& \mathrm{Ch}_{2}(a, \mathbf{k})=a^{2}+2 \hbar^{2}|\mathbf{k}|, \\
& \mathrm{Ch}_{3}(a, \mathbf{k})=a^{3}+6 \hbar^{2} a|\mathbf{k}|+3 \hbar^{3} \sum_{i} k_{i}\left(k_{i}+1-2 i\right), \quad \ldots .
\end{aligned}
$$

Правая часть последнего равенства содержит легко узнаваемое выражение (см., например, [12])

$$
\sum_{i} k_{i}\left(k_{i}+1-2 i\right)=\sum_{i}\left(\left(k_{i}-i+\frac{1}{2}\right)^{2}-\left(-i+\frac{1}{2}\right)^{2}\right)
$$

встречающееся в комбинаторике чисел Гурвица, - класс транспозиции, и это ровно тот самый элемент, присутствие которого при $t_{2}=1 / 2$ в (30) обеспечивает появление асимптотических чисел Гурвица через разложение функции Ламберта.

Дуальность между топологическими струнами и калибровочными теориями реинтерпретирует сумму по разбиениям в выражении для экспоненты от полного потенциала Громова-Виттена (33) как сумму по инстантонам в деформированной четырехмерной $\mathcal{N}=2$ суперсимметричной калибровочной теории [7]. Выражение (33) представляет собой частный случай статсуммы Некрасова [11] для $N_{c}=1$ или деформированной $U(1)$-калибровочной теории.

Формула (33) также утверждает, что квазиклассическое приближение $\hbar \rightarrow 0$ статсуммы Некрасова совпадает с потенциалом Громова-Виттена рода 0 или препотенциалом Виттена-Зайберга расширенной $U(1)$-теории. Эта эквивалентность приводит, в частности, к достаточно странному явлению: эффективные действия в четырехмерных суперсимметричных калибровочных теориях удовлетворяют тем же 
самым дифференциальным уравнениям, что и производящие функции корреляторов в топологических струнах!

Квазиклассический вклад в статсумму (33) может быть найден как решение задачи на экстремум для функционала

$$
\begin{aligned}
\mathcal{F}= & \frac{1}{2} \int d x f^{\prime \prime}(x) \sum_{k>0} t_{k} \frac{x^{k+1}}{k+1}-\frac{1}{2} \int_{x_{1}>x_{2}} d x_{1} d x_{2} f^{\prime \prime}\left(x_{1}\right) f^{\prime \prime}\left(x_{2}\right) F\left(x_{1}-x_{2}\right)+ \\
& +a^{D}\left(a-\frac{1}{2} \int d x x f^{\prime \prime}(x)\right)+\sigma\left(1-\frac{1}{2} \int d x f^{\prime \prime}(x)\right),
\end{aligned}
$$

форма которого определяется из интегрального представления для полиномов Чженя

$$
\mathrm{Ch}_{l}(a, \mathbf{k})=\frac{1}{2} \int d x f_{\mathbf{k}}^{\prime \prime}(x) x^{l} \sim \sum_{i=1}^{\infty}\left(\left(a+\hbar\left(k_{i}-i+1\right)\right)^{l}-\left(a+\hbar\left(k_{i}-i\right)\right)^{l}\right)
$$

и меры Планшереля

$$
\begin{aligned}
\mathbf{m}_{\mathbf{k}}^{2} & \sim \prod_{i, j}\left(k_{i}-k_{j}+j-i\right)=\exp \left(\sum_{i, j} \ln \left(k_{i}-k_{j}+j-i\right)\right) \sim \\
& \sim \exp \left(-\frac{1}{2 \hbar^{2}} \int_{x_{1}>x_{2}} d x_{1} d x_{2} f_{\mathbf{k}}^{\prime \prime}\left(x_{1}\right) f_{\mathbf{k}}^{\prime \prime}\left(x_{2}\right) \gamma\left(x_{1}-x_{2} ; \hbar\right)\right) \underset{\hbar \rightarrow 0}{\sim} \\
& \underset{\hbar \rightarrow 0}{\sim} \exp \left(-\frac{1}{2 \hbar^{2}} \int_{x_{1}>x_{2}} d x_{1} d x_{2} f_{\mathbf{k}}^{\prime \prime}\left(x_{1}\right) f_{\mathbf{k}}^{\prime \prime}\left(x_{2}\right) F\left(x_{1}-x_{2}\right)\right)
\end{aligned}
$$

через вторую производную профильной функции [13]

$$
f_{\mathbf{k}}^{\prime \prime}(x) \sim 2 \sum_{i=1}^{\infty}\left(\delta\left(x-a-\hbar\left(k_{i}-i+1\right)\right)-\delta\left(x-a-\hbar\left(k_{i}-i\right)\right)\right)
$$

для разбиений. В формуле $(40)$ ядро $\gamma(x ; \hbar)$ удовлетворяет разностному уравнению второго порядка

$$
\gamma(x+\hbar)+\gamma(x-\hbar)-2 \gamma(x)=\hbar^{2} \ln x
$$

и при $\hbar \rightarrow 0$ может быть в главном приближении заменено функцией

$$
\gamma(x ; \hbar) \underset{\hbar \rightarrow 0}{\Longrightarrow} F(x)=\frac{x^{2}}{2}\left(\ln x-\frac{3}{2}\right),
$$

удовлетворяющей уравнению $F^{\prime \prime}(x)=\ln x$ и известной как пертурбативный препотенциал в четырехмерной $\mathcal{N}=2$ суперсимметричной калибровочной теории.

Профильная функция

$$
f_{\mathbf{k}}(x)=|x-a|+\Delta f_{Y_{\mathbf{k}}}(x) \sim \sum_{i=1}^{\infty}\left(\left|x-a-\hbar\left(k_{i}-i+1\right)\right|-\left|x-a-\hbar\left(k_{i}-i\right)\right|\right)
$$


буквально отвечает профилю диаграммы Юнга $Y_{\mathbf{k}}$ разбиения $\mathbf{k}$, помещенной в прямой угол $|x-a|$, вершина которого находится в точке $x=a$ на оси абсцисс. Функционал (38) должен быть вычислен на экстремальном разбиении $\mathbf{k}_{*}$, отвечающем "большой" диаграмме Юнга $Y_{\mathbf{k}_{*}}$ с профильной функцией $f_{\mathbf{k}_{*}}(x) \equiv f(x)$, являющейся решением уравнения на экстремум для функционала (38). Два последних члена в правой части формулы (38) отвечают добавленным с множителями Лагранжа дополнительным условиям на профильную функцию, следующим из (44), а именно равенству $f_{\mathbf{k}}^{\prime}\left(x^{+}\right)-f_{\mathbf{k}}^{\prime}\left(x^{-}\right)=2$, отвечающему "выходу" на прямой угол профильной функции $f_{\mathbf{k}}\left(x^{ \pm}\right)=\left|x^{ \pm}-a\right|$ при некоторых $x^{ \pm}$, и тому, что вершина диаграммы Юнга и прямого угла находится в точке $a=\frac{1}{2} \int d x x f_{\mathbf{k}}^{\prime \prime}(x)$.

Написав уравнения экстремума для функционала (38), получаем для $S(z)=$ $\frac{d}{d z} \frac{\delta \mathcal{F}}{\delta f^{\prime \prime}(z)}$, или

$$
S(z)=\sum_{k>0} t_{k} z^{k}-\int d x f^{\prime \prime}(x)(z-x)(\ln (z-x)-1)-a^{D},
$$

что вещественная часть (45) равна нулю на сегменте I,

$$
\operatorname{Re} S(z)=\frac{1}{2}(S(z+i 0)+S(z-i 0))=0, \quad z \in \mathbf{I},
$$

где $\Delta f(x) \neq 0: x^{-}<x<x^{+}$. Асимптотика выражения (45) при $z \rightarrow \infty$ совпадает с (16), и чтобы построить такую функцию, удовлетворяющую условию (46), надо взять двойное накрытие $z$-плоскости $y^{2}=\left(z-x^{+}\right)\left(z-x^{-}\right)$, эквивалентное цилиндру (14), где $x^{ \pm}=v \pm 2 \Lambda$, и написать нечетное относительно инволюции, меняющей местами два z-листа, выражение (17), автоматически удовлетворяющее условию (46).

Экстремальная профильная функция находится из выражения (17) как скачок $f^{\prime}(x) \sim \operatorname{jump}(d S / d x)$, что следует из интегрального представления (45). В работе [9], в частности, было найдено, что если добавить ненулевое время $t_{2} \neq 0$ к малому фазовому пространству, то экстремальная профильная функция равна

$$
\begin{gathered}
f^{\prime}(x)=\frac{2}{\pi}\left(\arcsin \left(\frac{x-v}{2 \Lambda}\right)+2 t_{2} \sqrt{4 \Lambda^{2}-(x-v)^{2}}\right), \\
v-2 \Lambda \leqslant x \leqslant v+2 \Lambda
\end{gathered}
$$

т.е. арксинус Вершика-Керова деформируется полукруговым распределением Вигнера и "перенормировкой" $v=a \rightarrow v(a ; \mathbf{t})$ и $\Lambda=e^{t_{1} / 2} \rightarrow \Lambda(a ; \mathbf{t})$ параметров кривой, являющихся решениями уравнений (18).

\section{4. ПОЛНЫЙ КВАЗИКЛАССИЧЕСКИЙ ПОТЕНЦИАЛ ГРОМОВА-ВИТТЕНА $\mathbb{P}^{1}$}

Чтобы восстановить зависимость от времен $\mathbf{T}$ в статсумме, $\tau(a, \mathbf{t}) \rightarrow \tau(a, \mathbf{t}, \mathbf{T})$, или чтобы включить потомки единичного оператора $\left\{\sigma_{k}(\mathbf{1})\right\}$ при $k>0$, следует найти решение условий Вирасоро

$$
L_{n}\left(\mathbf{t}, \mathbf{T} ; \partial_{\mathbf{t}}, \partial_{\mathbf{T}} ; \partial_{\mathbf{t}}^{2}\right) \tau(a, \mathbf{t}, \mathbf{T})=0, \quad n \geqslant-1,
$$


с начальным условием $\tau(a, \mathbf{t})=\left.\tau(a, \mathbf{t}, \mathbf{T})\right|_{T_{n}=\delta_{n, 1}}$ (см. [14]-[18]). В квазиклассическом приближении решение этих условий Вирасоро, приводящее к полному потенциалу Громова-Виттена рода $0 \mathcal{F}(a, \mathbf{t}, \mathbf{T})$, может быть описано [19] следующим обобщением формулы (16):

$$
S(z) \underset{z \rightarrow \infty}{=} \sum_{k>0} t_{k} z^{k}-2 \sum_{n>0} T_{n} z^{n}\left(\ln z-c_{n}\right)+2 a \ln z-\frac{\partial \mathcal{F}}{\partial a}-2 \sum_{k>0} \frac{1}{k z^{k}} \frac{\partial \mathcal{F}}{\partial t_{k}},
$$

где $c_{k}=\sum_{i=1}^{k} 1 / i$ обозначают гармонические числа. Функция (49) определяет $\mathcal{F}$, будучи построенной глобально на кривой (14), нечетной относительно инволюции $w \leftrightarrow 1 / w$. Для этого опять следует подставить $z^{k} \rightarrow \Omega_{k}(w)=z(w)_{+}^{k}-z(w)_{-}^{k}$ и

$$
z^{k}\left(\ln z-c_{k}\right) \rightarrow H_{k}(z, w)=z^{k} \ln w+\sum_{j=1}^{k} C_{j}^{(k)} \Omega_{j}(w)
$$

для полиномиальных t-потоков и логарифмических Т-потоков соответственно. Коэффициенты $C_{j}^{(k)}$ в правой части (50) полностью фиксируются асимптотикой при $z \rightarrow \infty$ (подробнее см. в [19]). Зависимость от времен $\mathbf{T}$ потенциала $\mathcal{F}(a, \mathbf{t}, \mathbf{T})$ определяется условиями

$$
\left.\frac{\partial \mathcal{F}}{\partial T_{n}}\right|_{\mathbf{t}}=(-1)^{n} n !\left(S_{n}\right)_{0}
$$

(наводящими соображениями для которых могут быть формулы Саито [20]), где

$$
\frac{d^{n} S_{n}}{d z^{n}}=S, \quad n \geqslant 0
$$

или $S_{n}$ является $n$-й первообразной $S$ (нечетной относительно инволюции $\left.w \leftrightarrow 1 / w\right)$. Естественно, что в частном случае $n=0$ формула (51) совпадает с (25), поскольку переменная $а$ отвечает примарному оператору $\sigma_{0}(\mathbf{1}) \equiv \mathbf{1}$. При $n=1$ формула $(51)$ приводит к выражению

$$
\mathcal{F}\left(t_{1}, a, T_{1}\right)=\frac{a^{2} t_{1}}{2 T_{1}}+T_{1}^{2} e^{t_{1} / T_{1}}
$$

которое при $T_{1}=1$, очевидно, совпадает с (10), в то время как при $T_{1} \rightarrow \infty$ дает

$$
\mathcal{F}\left(t_{1}, a, T_{1}\right) \underset{T_{1} \rightarrow \infty}{\sim} \cdots+\mathrm{F}_{\mathrm{K}}\left(t_{1}+a, T_{1}\right)+\mathrm{F}_{\mathrm{K}}\left(t_{1}-a, T_{1}\right)+\cdots
$$

т.е. то, с чего мы начали в (2), (7), - линейные по $x$ решения уравнения КдФ $u\left(x, T_{1}\right) \sim x / T_{1}$.

Зависимость от времен $\mathbf{t}$ квазиклассической тау-функции $\mathcal{F}(\mathbf{t}, \mathbf{T})$ определяется бездисперсионной иерархией цепочки Тоды, в то время как зависимость от времен $\mathbf{T}$ может быть описана в терминах так называемой расширенной иерархии Тоды [21], [18], являющейся весьма специальной нетривиальной редукцией [15], [17] двумеризованной решетки Тоды. На малом фазовом пространстве

$$
\mathcal{F}_{\text {eq }}\left(X_{1}, \bar{X}_{1} ; \epsilon\right)=\frac{\epsilon^{2}}{6}\left(X_{1}^{3}+\bar{X}_{1}^{3}\right)+e^{X_{1}+\bar{X}_{1}}=\frac{a^{2} t_{1}}{2}+e^{t_{1}}+\frac{\epsilon}{2} a t_{1}^{2}+\frac{\epsilon^{2}}{6} t_{1}^{3},
$$


что совпадает с (10) при $\epsilon \rightarrow 0$, при замене

$$
\begin{aligned}
t_{k} & =X_{k}+\bar{X}_{k}, & & k>0, \\
T_{k} & =-\epsilon \bar{X}_{k+1}, & & k \geqslant 0,
\end{aligned}
$$

или

$$
\frac{\partial}{\partial X_{k}}=\frac{\partial}{\partial t_{k}}, \quad \frac{\partial}{\partial \bar{X}_{k}}=\frac{\partial}{\partial t_{k}}-\epsilon \frac{\partial}{\partial T_{k-1}}, \quad k>0,
$$

для производных, действительно удовлетворяет двумеризованному уравнению Тоды

$$
\frac{\partial^{2} \mathcal{F}_{\mathrm{eq}}}{\partial \bar{X}_{1} \partial X_{1}}=\exp \left[\frac{1}{\epsilon^{2}}\left(\frac{\partial}{\partial X_{1}}-\frac{\partial}{\partial \bar{X}_{1}}\right)^{2} \mathcal{F}\right],
$$

если ограничиться решениями, удовлетворяющими редукции

$$
\frac{\partial \mathcal{F}_{\mathrm{eq}}}{\partial X_{1}}-\frac{\partial \mathcal{F}_{\mathrm{eq}}}{\partial \bar{X}_{1}}=\epsilon \frac{\partial \mathcal{F}_{\mathrm{eq}}}{\partial X_{0}}
$$

На малом фазовом пространстве формулы (56) дают просто $X_{1}=t_{1}+a / \epsilon$ и $\bar{X}_{1}=$ $-a / \epsilon$.

Уравнение кривой для эквивариантной решетки Тоды [15], которое недавно было выведено в рамках функционального подхода в работе [22], может быть записано в виде

$$
z=v+\Lambda\left(w+\frac{1}{w}\right)-\epsilon \ln \frac{z}{\Lambda w}
$$

и является деформацией кривой (14), но уже не алгебраической. Это уравнение следует дополнить сопряженным уравнением

$$
\bar{z}=\bar{v}+\Lambda\left(w+\frac{1}{w}\right)+\epsilon \ln \frac{\bar{z} w}{\Lambda},
$$

где параметры $v$ и $\bar{v}$ удовлетворяют условию редукции (59), или

$$
v-\bar{v}=\epsilon \ln \Lambda^{2} \text {. }
$$

Тем самым уравнения (60), (61) могут быть решены относительно $z=z(w)$ и $\bar{z}=$ $\bar{z}\left(w^{-1}\right)$ только в терминах разложений

$$
\begin{aligned}
& z \underset{w \rightarrow \infty}{=} \Lambda w+v+\left(\Lambda-\frac{\epsilon v}{\Lambda}\right) \frac{1}{w}+\cdots, \\
& \bar{z} \underset{w \rightarrow 0}{=} \frac{\Lambda}{w}+\bar{v}+\left(\Lambda+\frac{\epsilon \bar{v}}{\Lambda}\right) w+\cdots,
\end{aligned}
$$

которые дают, в частности, выражения для гамильтонианов двумеризованной цепочки Тоды

$$
h_{k}=z^{k}(w)_{+}+\frac{1}{2} z^{k}(w)_{0}, \quad \bar{h}_{k}=\bar{z}^{k}\left(w^{-1}\right)_{-}+\frac{1}{2} \bar{z}^{k}\left(w^{-1}\right)_{0},
$$


например

$$
\begin{aligned}
h_{1}(w) & =\Lambda w+\frac{v}{2}, \\
\bar{h}_{1}\left(w^{-1}\right) & =\frac{\Lambda}{w}+\frac{\bar{v}}{2}, \\
h_{2}(w) & =\Lambda^{2} w^{2}+2 \Lambda v w+\frac{v^{2}}{2}+\Lambda^{2}-\epsilon v, \\
\bar{h}_{2}\left(w^{-1}\right) & =\frac{\Lambda^{2}}{w^{2}}+\frac{2 \Lambda \bar{v}}{w}+\frac{\bar{v}^{2}}{2}+\Lambda^{2}+\epsilon \bar{v}, \ldots .
\end{aligned}
$$

Обратное к первому уравнению из (63) имеет вид

$$
-\ln \frac{w}{z}=\ln \Lambda+\frac{v}{z}+\cdots
$$

это означает, что

$$
\begin{aligned}
\ln \Lambda^{2} & =\frac{\partial^{2} \mathcal{F}_{\mathrm{eq}}}{\partial X_{0}^{2}}=X_{1}+\bar{X}_{1}, \\
v & =\frac{\partial^{2} \mathcal{F}_{\mathrm{eq}}}{\partial X_{0} \partial X_{1}}=\epsilon X_{1},
\end{aligned}
$$

a

$$
\bar{v}=v-\epsilon \ln \Lambda^{2}=-\epsilon \bar{X}_{1}=\frac{\partial^{2} \mathcal{F}_{\mathrm{eq}}}{\partial X_{0} \partial \bar{X}_{1}} .
$$

Решения (66) могут быть также найдены из уравнений (18) для производящей функции

$$
S_{\mathrm{eq}}=\sum_{k>0}\left(\frac{Y_{k}}{k} h_{k}(w)-\frac{\bar{Y}_{k}}{k} \bar{h}_{k}\left(w^{-1}\right)\right)-X_{0} \ln w
$$

где "точные" тодовские переменные $\left\{Y_{k}, \bar{Y}_{k}\right\}, k>0$, связаны с временами $\left\{X_{k}, \bar{X}_{k}\right\}$, определенными в (56), треугольным преобразованием [15], [17]. В частности,

$$
X_{1}=Y_{1}+1+\frac{X_{0}}{\epsilon}, \quad \bar{X}_{1}=\bar{Y}_{1}+1-\frac{X_{0}}{\epsilon},
$$

что автоматически приводит к выполнению условия редукции (59).

\section{5. ЭЛЛИПТИЧЕСКАЯ $U(1)$-ТЕОРИЯ}

Прежде чем перейти к неабелевым калибровочным теориям и отвечающим им пространственно-временным кривым старших родов, обсудим полезный эллиптический пример препотенциала, который, с одной стороны, является лишь непосредственным обобщением формулы (10) на малом фазовом пространстве, а с другой представляет собой один из немногих примеров того, как в нерациональном случае препотенциал может быть вычислен явно. Этот случай отвечает $U(1)$-калибровочной теории с массивной присоединенной материей, для которой пространственновременную кривую $\mathbb{P}^{1}$ следует заменить на эллиптическую кривую или комплексный тор с модулем $\tau$, играющим роль времени $t_{1}$ или ультрафиолетовой константы связи в калибровочной теории с нулевой бета-функцией. 
Рассмотрим эллиптическую кривую $\mathcal{E}_{\tau}=\mathbb{C} /(1, \tau)$ с отмеченной точкой $P$. Выберем $\xi(P)=0$, где $d \xi \in H^{1}\left(\mathcal{E}_{\tau}\right)$ представляет собой канонический голоморфный дифференциал

$$
\oint_{A} d \xi=1, \quad \oint_{B} d \xi=\tau,
$$

возникающий здесь вместо "голоморфного" абелева дифференциала третьего рода на цилиндре $(14) d \Phi=d w / w \rightarrow d \xi$. Рассмотрим также абелев дифференциал второго рода

$$
d \lambda=-M d \xi(\wp(\xi)+2 \eta)=-M \frac{d \xi}{\xi^{2}}+\cdots
$$

с константой $\eta=\zeta(1 / 2)$, выбранной с целью зафиксировать постоянство его периодов

$$
\oint_{A} d \lambda=0, \quad \oint_{B} d \lambda=-M\left(2 \eta \tau-2 \eta^{\prime}\right)=-2 \pi i M=\text { const }
$$

где $\eta^{\prime}=\zeta(\tau / 2)$ и второе равенство во второй формуле выполняется благодаря тождеству Лежандра. Проинтегрировав (71), получим

$$
\lambda=M(\zeta(\xi)-2 \eta \xi+i \pi)+a=M \frac{\theta^{\prime}(\xi)}{\theta(\xi)}+a+i \pi M
$$

где $\theta(\xi) \equiv \theta_{1}(\xi \mid \tau)$ обозначает единственную нечетную тета-функцию Якоби, а константа интегрирования выбрана так, чтобы получить

$$
\oint_{A} d S=\oint_{A} \lambda d \xi=a
$$

Для дуального $B$-периода имеем

$$
\oint_{B} d S=\oint_{B} \lambda d \xi=(a+i \pi M) \tau+M \ln \frac{\theta\left(\xi_{0}+\tau\right)}{\theta\left(\xi_{0}\right)}=a \tau+i \pi M\left( \pm 1-2 \xi_{0}\right),
$$

где $\xi_{0}$ обозначает точку пересечения $A$ - и $B$-циклов. Вычисляя производные производящей 1-формы, получим

$$
\begin{aligned}
& \frac{\partial}{\partial a} d S=\left.\frac{\partial \lambda}{\partial a}\right|_{\xi, M, \tau} d \xi=d \xi \\
& \frac{\partial}{\partial \tau} d S=\left.\frac{\partial \lambda}{\partial \tau}\right|_{\xi, M, a} d \xi=M\left(\frac{\partial}{\partial \tau} \ln \theta(\xi)\right)^{\prime} d \xi=4 \pi i M\left(\frac{\theta^{\prime \prime}(\xi)}{\theta(\xi)}\right)^{\prime} d \xi
\end{aligned}
$$

т.е. производная по $\tau$ приводит к появлению неоднозначного дифференциала, так как

$$
\begin{aligned}
& \Omega=\frac{\theta^{\prime \prime}(\xi)}{\theta(\xi)}=(\ln \theta(\xi))^{\prime \prime}+\left((\ln \theta(\xi))^{\prime}\right)^{2} \equiv J^{\prime}(\xi)+J^{2}(\xi), \\
& \Omega\left(\xi_{0}+1\right)-\Omega\left(\xi_{0}\right)=0, \\
& \Omega\left(\xi_{0}+\tau\right)-\Omega\left(\xi_{0}\right)=\left(J\left(\xi_{0}+\tau\right)-J\left(\xi_{0}\right)\right)\left(J\left(\xi_{0}+\tau\right)+J\left(\xi_{0}\right)\right)= \\
&=-4 \pi i\left(J\left(\xi_{0}\right)-i \pi\right)=-4 \pi i\left(\frac{\theta\left(\xi_{0}\right)^{\prime}}{\theta\left(\xi_{0}\right)}-i \pi\right),
\end{aligned}
$$


и поэтому скачок (ср., например, с [10], [23])

$$
\Delta_{A} d \Omega=d \Omega\left(\xi_{0}+\tau\right)-d \Omega\left(\xi_{0}\right)=-4 \pi i d\left(\frac{\theta\left(\xi_{0}\right)^{\prime}}{\theta\left(\xi_{0}\right)}\right)=-\frac{4 \pi i}{M} d \lambda .
$$

Чтобы восстановить нетривиальную часть препотенциала, следует теперь вычислить

$$
\frac{\partial \mathcal{F}}{\partial \tau}=\frac{1}{2} \oint_{A} \lambda^{2} d \xi=\frac{1}{2}\left(a^{2}+\pi^{2} M^{2}+M^{2} \oint_{A}\left(\frac{\theta(\xi)^{\prime}}{\theta(\xi)}\right)^{2} d \xi\right)
$$

Для последнего интеграла имеем

$$
\begin{aligned}
\oint_{A}\left(\frac{\theta(\xi)^{\prime}}{\theta(\xi)}\right)^{2} d \xi= & \pi^{2} \int_{\xi_{0}}^{\xi_{0}+1} \operatorname{ctg}^{2}(\pi \xi) d \xi+ \\
& +8 \pi^{2} \sum_{n>0} \frac{q^{2 n}}{1-q^{2 n}} \int_{\xi_{0}}^{\xi_{0}+1} \operatorname{ctg}(\pi \xi) \sin (2 \pi n \xi) d \xi+ \\
& +16 \pi^{2} \sum_{n, k>0} \frac{q^{2 n}}{1-q^{2 n}} \frac{q^{2 k}}{1-q^{2 k}} \int_{\xi_{0}}^{\xi_{0}+1} \sin (2 \pi n \xi) \sin (2 \pi k \xi) d \xi
\end{aligned}
$$

где $q=e^{i \pi \tau}$ и

$$
\begin{aligned}
\int_{\xi_{0}}^{\xi_{0}+1} \operatorname{ctg}^{2}(\pi \xi) d \xi & =-\pi^{2}, \\
\int_{\xi_{0}}^{\xi_{0}+1} \operatorname{ctg}(\pi \xi) \sin (2 \pi n \xi) d \xi & =1, \quad n>0, \\
\int_{\xi_{0}}^{\xi_{0}+1} \sin (2 \pi n \xi) \sin (2 \pi k \xi) d \xi & =\frac{1}{2} \delta_{n, k}, \quad n, k>0 .
\end{aligned}
$$

Поэтому получаем

$$
\frac{\partial \mathcal{F}}{\partial \tau}=\frac{1}{2} \oint_{A} \lambda^{2} d \xi=\frac{a^{2}}{2}+4 \pi^{2} M^{2} \sum_{n>0} \frac{q^{2 n}}{\left(1-q^{2 n}\right)^{2}} .
$$

Интегрируя это соотношение, наконец, получим

$$
\mathcal{F}=\frac{\tau a^{2}}{2}+\frac{2 \pi M^{2}}{i} \sum_{n>0} \int \frac{q^{2 n-1} d q}{\left(1-q^{2 n}\right)^{2}}=\frac{\tau a^{2}}{2}+\frac{\pi M^{2}}{i} \sum_{n>0} \frac{1}{n}\left(\frac{1}{1-q^{2 n}}-1\right) .
$$

Пересуммирование последнего выражения дает

$$
\sum_{n>0} \frac{1}{n}\left(\frac{1}{1-q^{2 n}}-1\right)=\sum_{n, k>0} \frac{q^{2 n k}}{n}=-\sum_{k>0} \ln \left(1-q^{2 k}\right),
$$

так что

$$
\mathcal{F}=\frac{1}{2} \tau a^{2}-m^{2} \ln \prod_{k>0}\left(1-q^{2 k}\right) \sim \frac{1}{2} \tau a^{2}-m^{2} \ln \eta(\tau)
$$


где введена физическая масса $m^{2}=(\pi / i) M^{2}$, а $\eta(\tau)=q^{1 / 12} \prod_{k>0}\left(1-q^{2 k}\right)$ обозначает функцию Дедекинда (выбрасывание или добавление к препотенциалу линейного члена $\ln q \sim \tau$ несущественно). В пределе $m \rightarrow \infty, \tau \rightarrow+i \infty$ при условии

$$
m^{2} e^{2 \pi i \tau}=\Lambda^{2}=e^{t_{1}}=\text { fixed }
$$

мы возвращаемся от (85) к формуле (10) (опять же, вообще говоря, с точностью до несущественной максимально квадратичной формы). Довольно необычным фактом является появление функции Дедекинда $\eta(q)$ в основном квазиклассическом вкладе или члене с $g=0$ в разложении по родам производящей функции $\ln \tau=\hbar^{-2} \mathcal{F}+$ $F_{1}+\hbar^{2} F_{2}+\cdots$, в то время как обычно такой вклад естественным образом возникает в $F_{1} \sim \ln \operatorname{det} \bar{\partial}$. Это явление может быть связано с нетривиальными вопросами, касающимися модулярных свойств $F_{g}$ и уравнений голоморфной аномалии [24], [25], [7], обсуждение которых выходит за рамки настоящей работы.

\section{6. НЕАБЕЛЕВА ТЕОРИЯ}

Дуальность между топологическими струнами и калибровочными теориями утверждает, в частности, что редуцированный потенциал Громова-Виттена рода 0 $\mathcal{F}(a, \mathbf{t})$ совпадает с частным упрощенным случаем $N_{c}=1$ препотенциала (расширенной) $\mathcal{N}=2$ суперсимметричной калибровочной теории Виттена-Зайберга. Более того, оказывается, что квазиклассическое решение неабелевой теории с калибровочной группой $U\left(N_{c}\right)$ может быть получено практически тем же путем [9], а именно путем нахождения экстремальных решений для функционала (38), с единственным небольшим изменением дополнительных связей в задаче. Вместо единственного условия $1 / 2 \int d x x f^{\prime \prime}(x)=a$ теперь следует рассматривать $N_{c}$ аналогичных условий

$$
\frac{1}{2} \int_{\mathbf{I}_{i}} d x x f^{\prime \prime}(x)=a_{i}, \quad i=1,2, \ldots, N_{c},
$$

на профильные функции $f(x)$, отвечающие $N_{c}$-кратным разбиениям [13], с вершинами в $a_{1}, \ldots, a_{N_{c}}$ соответственно. Все эти условия учитываются, как и в формуле (38), добавлением к функционалу с множителями Лагранжа $a_{1}^{D}, \ldots, a_{N_{c}}^{D}$.

Чтобы решить уравнения экстремума при наличии набора новых условий, рассматривается двойное накрытие $z$-плоскости с $N_{c}$ разрезами $\left\{\mathbf{I}_{j}: x_{j}^{-}<z<x_{j}^{+}\right\}$

$$
y^{2}=\prod_{i=1}^{N_{c}}\left(z-x_{i}^{+}\right)\left(z-x_{i}^{-}\right)
$$

или гиперэллиптическая кривая рода $N_{c}-1$ и строится функция $S$, нечетная относительно инволюции $y \leftrightarrow-y$ или чисто мнимая на объединении $\mathbf{I}$ этих $N_{c}$ разрезов: $\mathbf{I}=\bigcup_{j=1}^{N_{c}} \mathbf{I}_{j}$.

Такая неабелева расширенная $U\left(N_{c}\right) \mathcal{N}=2$ суперсимметричная калибровочная теория решается с помощью абелевых дифференциалов. Функциональное уравнение (46) решается теперь путем построения дифференциала от

$$
\Phi(z)=\frac{d S}{d z}=\sum_{k>0} k t_{k} z^{k-1}-\frac{1}{2} \int d x f^{\prime \prime}(x) \ln (z-x)
$$


(вспомним, что производная профильной функции восстанавливается из скачка $\left.f^{\prime}(x) \sim \operatorname{jump} \Phi(x)\right)$. На гиперэллиптической кривой (88) для дифференциала $d \Phi$ имеем

$$
d \Phi= \pm \frac{\phi(z) d z}{y}= \pm \frac{\phi(z) d z}{\sqrt{\prod_{i=1}^{N_{c}}\left(z-x_{i}^{+}\right)\left(z-x_{i}^{-}\right)}},
$$

где числитель $\phi(z)$ полностью фиксируется асимптотикой и периодами

$$
\oint_{A_{j}} d \Phi=-2 \pi i \int_{\mathbf{I}_{j}} f^{\prime \prime}(x) d x=-2 \pi i\left(f^{\prime}\left(x_{j}^{+}\right)-f^{\prime}\left(x_{j}^{-}\right)\right)=-4 \pi i .
$$

Если все $t_{k}=0, k>1, t_{1}=\ln \Lambda^{N_{c}}$ (конечно, по-прежнему $\left.T_{n}=\delta_{n, 1} !\right)$ в окрестности точек $P_{ \pm}$, для которых $z\left(P_{ \pm}\right)=\infty$, находим

$$
\Phi \underset{P \rightarrow P_{ \pm}}{=} \mp 2 N_{c} \ln z \pm 2 N_{c} \ln \Lambda+O\left(z^{-1}\right)
$$

и это означает, что существует мероморфная функция $w=\Lambda^{N_{c}} e^{-\Phi}$, удовлетворяющая уравнению (15). При $N_{c}=1$ мы воспроизводим кривую (14), или оператор Лакса бездисперсионной цепочки Тоды.

Если, однако, старшие времена $t_{k}$ не равны нулю, функция $e^{-\Phi}$ приобретает существенную особенность и не может быть описана алгебраически. Неявно все по-прежнему фиксируется асимптотикой

$$
d \Phi \underset{z \rightarrow \infty}{\sim} \sum_{k>1} k(k-1) t_{k} z^{k-2}+\cdots
$$

и условиями на периоды (91), обеспечивающими, в частности, обобщенный аналог условий (18)

$$
\delta(d S)=\delta(\Phi d z) \underset{z \rightarrow x_{j}^{ \pm}}{=} \frac{-\phi\left(x_{j}^{ \pm}\right) \delta x_{j}^{ \pm}}{\prod_{k}^{\prime} \sqrt{\left(x_{j}^{ \pm}-x_{k}^{+}\right)\left(x_{j}^{ \pm}-x_{k}^{-}\right)}} \frac{d z}{\sqrt{z-x_{j}^{ \pm}}}+\cdots \simeq \text { holomorphic. }
$$

Условия на $A$-периоды совместно с асимптотикой (93) полностью фиксируют форму дифференциала $d \Phi$. Равенство же нулю $B$-периодов вместе с целочисленностью коэффициента при логарифме в формуле (92) налагает $N_{c}$ условий на $2 N_{c}$ точек ветвления кривой (88). Оставшиеся $N_{c}$ неизвестных поглощаются периодами Виттена-Зайберга

$$
a_{i}=\frac{1}{4 \pi i} \oint_{A_{i}} z d \Phi, \quad i=1,2, \ldots, N_{c},
$$

куда мы также включили дополнительный $N_{c}$-й зависимый период; альтернативная возможность - зафиксировать вычет $а$ в бесконечности, как в формуле (24). Дуальные к (95) B-периоды определяют градиенты препотенциала

$$
a_{i}^{D}=\frac{1}{2} \oint_{B_{i}} z d \Phi=\frac{\partial \mathcal{F}}{\partial a_{i}}, \quad i=1,2, \ldots, N_{c}
$$


и, как и в $U(1)$-случае, производные по временам $\mathbf{t}$ определяются формулами вычетов

$$
\frac{\partial \mathcal{F}}{\partial t_{k}}=-\left.\frac{1}{k+1} \operatorname{res}\right|_{z=P_{+}}\left(z^{k+1} d \Phi\right), \quad k>0,
$$

но уже на кривой (88). Условие интегрируемости (22) для градиентов (96) обеспечивается симметричностью матрицы периодов кривой (88) или в более общем случае, включающем и формулы (97), билинейными соотношениями Римана для всех абелевых дифференциалов.

Если же старшие времена $T_{n}$ не равны нулю, скажем в ситуации, когда включены $N$ первых потомков единичного оператора с $n=1, \ldots, N^{1)}$, то лишь $(N+1)$-я производная от (49)

$$
d \Phi^{(N-1)}=d\left(\frac{d^{N} S}{d z^{N}}\right)
$$

может быть разложена по базису однозначных на кривой (88) абелевых дифференциалов. В этом случае снова все полностью фиксируется целочисленностью $A$-периодов и особенностями, но теперь уже не только при $z\left(P_{ \pm}\right)=\infty$, но и в точках ветвления $\left\{x_{j}\right\}, j=1,2, \ldots, 2 N_{c}$, кривой (88), где дифференциал (98) приобретает дополнительные полюсы. На самом деле эти дополнительные особенности "ненастоящие", в том смысле, что $\Phi^{\prime}, \ldots, \Phi^{(N-1)}$ регулярны в точках ветвления, если рассматривать их как 2-, $\ldots, N$-дифференциалы на кривой (88).

Чтобы построить дифференциал (98) явно, запишем

$$
d \Phi^{(N-1)}=\frac{\phi(z) d z}{y}+\frac{d z}{y} \sum_{j=1}^{2 N_{c}} \sum_{k=1}^{N-1} \frac{q_{j}^{k}}{\left(z-x_{j}\right)^{k}}
$$

и фиксируем периоды $d \Phi^{(N-1)}, d \Phi^{(N-2)}, \ldots, d \Phi^{\prime}, d \Phi$ с помощью $2 N_{c} \cdot N$ условий, оставаясь тем самым с

$$
(2 N+1) N_{c}-2 N_{c} \cdot N=N_{c}
$$

переменными, поглощаемыми обобщенными периодами Виттена-Зайберга

$$
a_{j}=\frac{1}{4 \pi i} \oint_{A_{j}} \frac{z^{N}}{N !} d \Phi^{(N-1)}, \quad j=1,2, \ldots, N_{c},
$$

которые по-прежнему определяют препотенциал с помощью формул

$$
a_{j}^{D}=\frac{1}{2} \oint_{B_{j}} \frac{z^{N}}{N !} d \Phi^{(N-1)}=\frac{\partial \mathcal{F}}{\partial a_{j}}, \quad j=1,2, \ldots, N_{c} .
$$

Обобщенная форма Виттена-Зайберга представляет собой теперь $N$-кратное преобразование Лежандра от функции $S$ (49), являющейся, конечно, многозначным абелевым интегралом на кривой (88).

1)В "минимальной” теории $T_{n}=\delta_{n, 1}$ и $\mathcal{F}=\mathcal{F}(a, \mathbf{t}) ; T_{1}=1$ отвечает ненулевому конденсату $\left\langle\sigma_{1}(\varpi)\right\rangle \neq 0$. 


\section{7. ДВЕ ФУНКЦИОНАЛЬНЫЕ ФОРМУЛИРОВКИ}

В пертурбативном пределе $\Lambda \rightarrow 0$ разрезы на кривой (88) сжимаются в точки $z=v_{j}, j=1, \ldots, N_{c}$, и кривая вырождается в рациональную, которую можно запараметризовать в виде

$$
w_{\text {pert }}=P_{N_{c}}(z)=\prod_{i=1}^{N_{c}}\left(z-v_{i}\right)
$$

Эта кривая оснащена теперь двумя полиномами (произвольной степени): $\mathbf{t}(z) \equiv$ $\sum_{k>0} t_{k} z^{k}$ и $T(x) \equiv \sum_{n>0} T_{n} x^{n}$. В этих условиях функция $S$ вычисляется явно и представляется выражением

$$
S(z)=\mathbf{t}^{\prime}(z)-2 \sum_{j=1}^{N_{c}} \sigma\left(z ; v_{j}\right)=\mathbf{t}^{\prime}(z)-2 \sum_{j=1}^{N_{c}} \sum_{k>0} \frac{T^{(k)}\left(v_{j}\right)}{k !}\left(z-v_{j}\right)^{k}\left(\ln \left(z-v_{j}\right)-c_{k}\right)
$$

т.е. полностью определяется с помощью функции

$$
\sigma(z ; x)=\sum_{k>0} \frac{T^{(k)}(x)}{k !}(z-x)^{k}\left(\ln (z-x)-c_{k}\right),
$$

где сумма конечна, если ограничиться $N$-м классом фоновых полей, когда только первые времена $T_{1}, \ldots, T_{N}$ не равны нулю.

Пертурбативный препотенциал определяется градиентами

$$
a_{i}^{D}=S\left(v_{i}\right)=\frac{\partial \mathcal{F}_{\mathrm{pert}}}{\partial a_{i}}
$$

и эта формула приводит к следующему явному выражению:

$$
\begin{gathered}
\mathcal{F}_{\text {pert }}\left(a_{1}, \ldots, a_{N_{c}} ; \mathbf{t}, \mathbf{T}\right)=\sum_{j=1}^{N_{c}} F_{\mathrm{UV}}\left(a_{j} ; \mathbf{t}, \mathbf{T}\right)+\sum_{i \neq j} F\left(a_{i}, a_{j} ; \mathbf{T}\right), \\
a_{j}=T\left(v_{j}\right), \quad j=1,2, \ldots, N_{c}
\end{gathered}
$$

где

$$
\begin{array}{r}
F_{\mathrm{UV}}(x) \equiv F_{\mathrm{UV}}(x ; \mathbf{t}, \mathbf{T})=\int_{0}^{x} \mathbf{t}^{\prime}(\mathrm{x}) d T(\mathbf{x}), \\
\frac{\partial^{2}}{\partial x_{1} \partial x_{2}} F\left(x_{1}, x_{2} ; \mathbf{T}\right)=T^{\prime}\left(x_{1}\right) T^{\prime}\left(x_{2}\right) \ln \left(x_{1}-x_{2}\right) .
\end{array}
$$

При $T_{n}=\delta_{n, 1}$ из формулы (108) следует, что

$$
\left.F_{\mathrm{UV}}(x)\right|_{T_{n}=\delta_{n, 1}}=\sum_{k>0} t_{k} \frac{x^{k+1}}{k+1}
$$

что является ультрафиолетовым препотенциалом теории при выключенных потомках единичного оператора, за исключением конденсата $\left\langle\sigma_{1}(\mathbf{1})\right\rangle$, а также

$$
\left.F\left(x_{1}, x_{2} ; \mathbf{T}\right)\right|_{T_{n}=\delta_{n, 1}}=F\left(x_{1}-x_{2}\right),
$$


где правая часть равенства зависит только от разности аргументов и не зависит от переменных $\mathbf{T}$, воспроизводя тем самым постоянное ядро из правой части формулы (43). В общем случае включение потомков единицы индуцирует репараметризацию в пространстве модулей от $a=v$ к $a=T(v)=\sum_{n} T_{n} v^{n}$, что также приводит к критичным усложнениям в формулах (108).

Пертурбативный препотенциал (107) определяет, таким образом, общий вид функционала $\mathcal{F}(\mathbf{a}, \mathbf{t}, \mathbf{T})$ следующей формулой:

$$
\begin{aligned}
\mathcal{F}= & -\frac{1}{2} \int_{x_{1}>x_{2}} d x_{1} d x_{2} f^{\prime \prime}\left(x_{1}\right) f^{\prime \prime}\left(x_{2}\right) F\left(x_{1}, x_{2} ; \mathbf{T}\right)+\int d x f^{\prime \prime}(x) F_{\mathrm{UV}}(x ; \mathbf{t}, \mathbf{T})+ \\
& +\sum_{i} a_{i}^{D}\left(a_{i}-\frac{1}{2} \int d x x f^{\prime \prime}(x)\right)+\sigma\left(1-\frac{1}{2} \int d x f^{\prime \prime}(x)\right) .
\end{aligned}
$$

Функционалом (111) можно пользоваться, за исключением одной ситуации, которую мы специально рассмотрим ниже, так же, как и функционалом (38) при $T_{n}=\delta_{n, 1}$. Например, поскольку ядро (вторая формула в (108)) не зависит от параметров $\mathbf{t}$, легко находим, что производные

$$
\frac{\partial \mathcal{F}}{\partial t_{k}}=\int d x f^{\prime \prime}(x) \frac{\partial F_{\mathrm{UV}}(x ; \mathbf{t}, \mathbf{T})}{\partial t_{k}}
$$

по-прежнему определяются "регулярным хвостом" разложения

$$
S(z)=\frac{d}{d z} \frac{\delta \mathcal{F}}{\delta f^{\prime \prime}(z)}=\mathbf{t}^{\prime}(z)-a^{D}-\int d x f^{\prime \prime}(x) \sigma(z ; x) .
$$

Однако для функционала (111) серьезной проблемой является вычисление производных $\partial \mathcal{F} / \partial T_{n}$, поскольку ядро $F\left(x_{1}, x_{2} ; \mathbf{T}\right)$ явно зависит от времен T. К счастью, существует эквивалентная альтернативная функциональная формулировка, связанная с функционалом (111) интегральным преобразованием

$$
\int d x \rho(x) g(x)=\int d x f^{\prime \prime}(x) \hat{D}_{N-1}(x) g(x),
$$

так что

$$
\begin{aligned}
\mathcal{F}=\mathcal{F}_{N}[\rho]= & \frac{1}{2} \int d x \rho(x) \mathbf{t}_{N}(x)+\frac{(-1)^{N}}{(2 N) !} \int d x_{1} d x_{2} \rho\left(x_{1}\right) \rho\left(x_{2}\right) H_{2 N}^{(+)}\left(x_{1}-x_{2}\right)+ \\
& +\sum_{n=0}^{N} \sigma_{n}\left(T_{n}-\frac{(-1)^{n-1}}{2(N-n) !} \int d x x^{N-n} \rho(x)\right),
\end{aligned}
$$

где

$$
\mathbf{t}_{N}(x)=\sum_{k>0} t_{k} \frac{x^{k+N}}{(k+1) \ldots(k+N)},
$$

ядро

$$
\frac{(-1)^{N-1}}{(2 N) !} H_{2 N}^{(+)}(x)=\frac{1}{(2 N) !} x^{2 N}\left(\ln x-c_{2 N}\right)
$$


не зависит от $\mathbf{T}$, а

$$
\widehat{D}_{N-1}(x)=T^{\prime}(x) \frac{d^{N-1}}{d x^{N-1}}-T^{\prime \prime}(x) \frac{d^{N-2}}{d x^{N-2}}+\cdots+(-1)^{N-1} T^{(N)}
$$

является дифференциальным оператором $(N-1)$-го порядка.

Нетривиальным следствием существования альтернативной функциональной формулировки (114) является формула Саито (51) для многократных первообразных

$$
\frac{\partial \mathcal{F}}{\partial T_{n}}=\sigma_{n}=(-1)^{n} n !\left(S_{n}\right)_{0}, \quad n=0,1, \ldots, N,
$$

в которой правая часть выражает множители Лагранжа из формулы (114) в терминах постоянных частей последовательности функций

$$
\begin{aligned}
S_{N-1}(z)= & \mathbf{t}_{N-1}(z)-\frac{(-1)^{N-1}}{(2 N-1) !} \int d x \rho(x) H_{2 N-1}^{(+)}(z-x)+ \\
& +\sum_{n=0}^{N-1} \sigma_{n}(-1)^{n} \frac{z^{N-n-1}}{(N-n-1) !}, \quad \ldots, \\
S(z)= & \mathbf{t}^{\prime}(z)-\frac{(-1)^{N-1}}{N !} \int d x \rho(x) H_{N}^{(+)}(z-x)+\sigma_{0},
\end{aligned}
$$

вещественные части которых обращаются в нуль на разрезах.

\section{8. ЗАКЛЮЧЕНИЕ}

Мы продемонстрировали выше, что иногда вместо прямого решения сложных проблем в квантовой теории поля и теории струн ответы можно находить, решая довольно простые дифференциальные уравнения. Полезные для нужд калибровочных и струнных теорий решения имеют достаточно странные свойства с традиционной точки зрения. Однако они по-прежнему связаны с геометрией комплексных кривых, а поэтому во многих случаях, в отличие от ситуации с комплексными многообразиями старших размерностей, могут быть описаны и посчитаны явно.

Упрощенная $N_{c}=1$ расширенная теория Громова-Виттена дуальна топологической струне типа А, или теории Громова-Виттена проективной прямой $\mathbb{P}^{1}$. Такая абелева $N_{c}=1$ теория решается полностью с помощью бездисперсионной расширенной иерархии цепочки Тоды, и это решение характеризуется неустранимым присутствием топологического члена Егучи-Янга. В общем случае полного потенциала Громова-Виттена мы получаем решение так называемой расширенной иерархии цепочки Тоды (расширенной потоками, отвечающими включению гравитационных потомков единичного оператора). Расширенную иерархию цепочки удобно рассматривать как предельный случай так называемой эквивариантной решетки Тоды весьма специальной редукции иерархии двумеризованной решетки Тоды. Выше мы описали явный вид геометрии этой редукции на малом фазовом пространстве явным образом.

Для того чтобы расширить эти результаты на случай неабелевых калибровочных теорий, необходимо применение техники построения квазиклассических тау-функций с помощью абелевых интегралов на гиперэллиптических кривых произвольного 
рода $N_{c}-1$. Мы продемонстрировали, как это вычисление может быть проведено явно на эллиптической кривой и как оно приводит к прямому обобщению решения цепочки Тоды на малом фазовом пространстве с помощью модулярной функции Дедекинда, а также привели общую неявную формулировку для старших родов. Необходимо отметить, что расширение теории Виттена-Зайберга, аналогичное включению всех гравитационных потомков, требует расширения базиса абелевых дифференциалов, имеющих дополнительные полюсы в точках ветвления кривой. Мы обсудили также некоторые тонкости функциональной формулировки в расширенном случае и предложили интегральное преобразование, связывающее две разные формы функционала, удобные для получения ответов на те или иные вопросы.

Благодарности. Автор благодарен А. Александрову, М. Казаряну, И. Кричеверу и, в особенности, Н. Некрасову за чрезвычайно полезные обсуждения. Работа была выполнена при частичной поддержке Федерального агентства по атомной энергии, РФФИ (грант № 08-01-00667), Программы поддержки ведущих научных школ (грант НШ-1615.2008.2), INTAS (грант № 05-1000008-7865), проекта ANR-05-BLAN-0029-01, программы NWO-РФФИ (грант № 047.017.2004.015), Российско-итальянской программы РФФИ (грант № 06-01-92059-СЕ) и фонда Династия.

\section{Список литературы}

[1] E. Witten, Nucl. Phys. B, 340:2-3 (1990), 281-332; R. Dijkgraaf, E. Witten, Nucl. Phys. B, 342:3 (1990), 486-522.

[2] V. Kac, A. Schwarz, Phys. Lett. B, 257:3-4 (1991), 329-334.

[3] М.Л. Концевич, Функи. анализ и его прил., 25:2 (1991), 50-57; Comm. Math. Phys., 147:1 (1992), 1-23.

[4] S. Kharchev, A. Marshakov, A. Mironov, A. Morozov, A. Zabrodin, Phys. Lett. B, 275:3-4 (1992), 311-314; arXiv: hep-th/9111037; Nucl. Phys. B, 380:1-2 (1992), 181-240; arXiv: hep-th/9201013.

[5] M. Fukuma, H. Kawai, R. Nakayama, Internat. J. Modern Phys. A, 6:8 (1991), 1385-1406;

R. Dijkgraaf, E. Verlinde, H. Verlinde, Nucl. Phys. B, 348:3 (1991), 435-456.

[6] М. Тода, Теория нелинейных решеток, Мир, М., 1984.

[7] A.S. Losev, A. B. Marshakov, N.A. Nekrasov, "Small instantons, little strings and free fermions", From Fields to Strings: Circumnavigating Theoretical Physics, Ian Kogan Memorial Collection, V. 1, eds. M. Shifman, A. Vainshtein, J. Wheater, World Scientific, Singapore, 2005, 581-621; arXiv: hep-th/0302191.

[8] A. Gorsky, I. Krichever, A. Marshakov, A. Mironov, A. Morozov, Phys. Lett. B, 355:3-4 (1995), 466-474; arXiv: hep-th/9505035.

[9] A. V. Marshakov, N. A. Nekrasov, JHEP, 2007, № 1, 104; arXiv: hep-th/0612019; A. В. Маршаков, ТМФ, 154:3 (2008), 424-450; arXiv: 0706.2857.

[10] I. M. Krichever, Comm. Pure Appl. Math., 47:4 (1994), 437-475; arXiv: hep-th/9205110.

[11] N. A. Nekrasov, Adv. Theor. Math. Phys., 7:5 (2003), 831-864; arXiv: hep-th/0206161.

[12] A. Okounkov, Math. Res. Lett., 7:4 (2000), 447-453; arXiv: math. AG/0004128.

[13] N. A. Nekrasov, A. Okounkov, "Seiberg-Witten theory and random partitions", The Unity of Mathematics, Progr. Math., 244, eds. P. Etingof, V. Retakh, I. M. Singer, Birkhäuser, Boston, MA, 2006, 525-596; arXiv: hep-th/0306238.

[14] T. Eguchi, K. Hori, S. K. Yang, Internat. J. Modern Phys. A, 10:29 (1995), 4203-4224; arXiv: hep-th/9503017.

3 Теоретическая и математическая физика, т. 159, № 2, 2009 г. 
[15] E. Getzler, Publ. Res. Inst. Math. Sci., 40:2 (2004), 507-536; arXiv: math.AG/0207025; arXiv: math.AG/0209110.

[16] A. Givental, Mosc. Math. J., 1:4 (2001), 551-568; arXiv: math.AG/0108100.

[17] A. Okounkov, R. Pandharipande, Ann. of Math. (2), 163:2 (2006), 517-560; arXiv: math.AG/0204305; The equivariant Gromov-Witten theory of $P^{1}$, arXiv: math. AG/0207233.

[18] G. Carlet, B. Dubrovin, Y. Zhang, Mosc. Math. J., 4:2 (2004), 313-332; arXiv: nlin.SI/0306060; B. Dubrovin, Y. Zhang, Comm. Math. Phys., 250:1 (2004), 161-193; arXiv: math.DG/0308152.

[19] A. Marshakov, JHEP, 3 (2008), 055; arXiv: 0712.2802.

[20] K. Saito, On the periods of primitive integrals, Preprint, Harvard Univ., Cambridge, MA, 1980; А. С. Лосев, ТMФ, 95:2 (1993), 307-316; arXiv: hep-th/9211090; T. Eguchi, H. Kanno, Y. Yamada, S.-K. Yang, Phys. Lett. B, 305:3 (1993), 235-241; arXiv: hep-th/9302048.

[21] T. Eguchi, S.-K. Yang, Modern Phys. Lett. A, 9:31 (1994), 2893-2902; arXiv: hep-th/9407134.

[22] A. Marshakov, Non Abelian gauge theories, prepotentials and Abelian differentials, arXiv: 0810.1536.

[23] H. W. Braden, A. Marshakov, A. Mironov, A. Morozov, Acta Appl. Math., 99:3 (2007), 223-244; arXiv: hep-th/0606035.

[24] M. Bershadsky, S. Cecotti, H. Ooguri, C. Vafa, S. Katz, Nucl. Phys. B, 405:2-3 (1993), 279-304; arXiv: hep-th/9302103; M. Bershadsky, S. Cecotti, H. Ooguri, C. Vafa, Comm. Math. Phys., 165:2 (1994), 311-427; arXiv: hep-th/9309140.

[25] A. Klemm, M. Mariño, S. Theisen, JHEP, 3 (2003), 051; arXiv: hep-th/0211216; R. Dijkgraaf, A. Sinkovics, M. Temürhan, Adv. Theor. Math. Phys., 7:6 (2003), 1155-1174; arXiv: hep-th/0211241; M. Aganagic, V. Bouchard, A. Klemm, Comm. Math. Phys., 277:3 (2008), 771-819; arXiv: hep-th/0607100. 